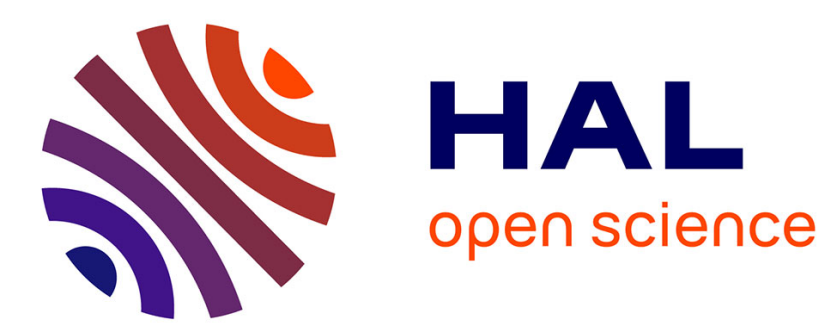

\title{
New Low-Temperature Thermistors InSb:Mn for Nuclear Cryogenic Detectors
}

\author{
S. Obukhov, V. Trofimov
}

\section{To cite this version:}

S. Obukhov, V. Trofimov. New Low-Temperature Thermistors InSb:Mn for Nuclear Cryogenic Detectors. Journal de Physique IV Proceedings, 1996, 06 (C3), pp.C3-169-C3-174. 10.1051/jp4:1996326 . jpa-00254244

\section{HAL Id: jpa-00254244 https://hal.science/jpa-00254244}

Submitted on 1 Jan 1996

HAL is a multi-disciplinary open access archive for the deposit and dissemination of scientific research documents, whether they are published or not. The documents may come from teaching and research institutions in France or abroad, or from public or private research centers.
L'archive ouverte pluridisciplinaire HAL, est destinée au dépôt et à la diffusion de documents scientifiques de niveau recherche, publiés ou non, émanant des établissements d'enseignement et de recherche français ou étrangers, des laboratoires publics ou privés. 


\title{
New Low-Temperature Thermistors InSb:Mn for Nuclear Cryogenic Detectors
}

\author{
S.A. Obukhov and V.N. Trofimov*
}

A.F. Ioffe Institute of Physics and Technology, Polytechnicheskaya 26, 194021, St.-Petersburg, Russia

* Joint Institute for Nuclear Research, Dubna, 141980, Russia

\begin{abstract}
InSb doped with $\mathrm{Mn}$ offers an excellent integrated cryogenic detector for low-energy X-ray and beta spectroscopy. It combines good properties from both spectroscopic point of view (high atomic number) and lowtemperature (LT) thermometry. The thermosensitivity is superiour over that of the most popular another bulk thermistor from neutron-transmutation-doped (NTD) Ge and its application is not restricted by the severe technological aspects. The results of (magneto)resistivity versus temperature $R(T)$ and magnetic susceptibility $\chi(T)$ measurements, performed to clear up an unusial behaviour of conductivity at $\mathrm{mK}$ temperatures, are presented .
\end{abstract}

\section{INTRODUCTION}

As is now commonly recognized, the cryogenic detectors of nuclear particles provide higher energy resolution and threshold compaired with the conventional, including semiconducting ones [1]. These principal advantages follow from the lower energy of elementary excitations, which are phonons instead of electron-hole pairs. The ultimate resolution of a microcalorimeter is limited by the thermodynamic temperature noise and is equal to $\delta \mathrm{E}=\sqrt{\mathrm{kB}_{\mathrm{B}}} 2 \mathrm{C}$, where $\mathrm{kB}$ is Boltzmann's constant, $\mathrm{T}$ is a temperature, and $\mathrm{C}$ is a heat capacity of the device. This gives, for example, the resolution of $1 \mathrm{eV}$ at appropriate mass $(\approx$ $1 \mathrm{mg})$ and temperature $(\approx 10 \mathrm{mK})$. In practice other factors limit the resolution at some higher level. As a rule, the smaller detector, the smaller difference between the theoretically expected and realized characteristics. A typical composite cryogenic detector includes an energy absorber ( high Debye temperature single crystals like sapphire, germanium, silicon, etc), and a temperature-to-voltage pulse transducer (semiconducting thermistors, supercoducting thin film thermometers, etc. ). It is cooled down in a dilution refrigerator to $\mathrm{mK}$ temperature range, when the heat capacity of the detector $\mathrm{C}$ drops to a very small value and the temperature change $\Delta T=E / C$ induced by the particle with energy $E$ can easily be measured. The most impressive results have been so far obtained for low-energy nuclear $\mathrm{X}$-ray spectrometry with the FWHM of $7.3 \mathrm{eV}$ for $6 \mathrm{keV}$ gammas from ${ }^{55} \mathrm{Fe}[2]$. The advantages of cryogenic detection over semiconducting were clearly demonstrated by the $\mathrm{X}$-ray spectra measured from one source through these two methods [3]. In this case the cryodetector was of composite type with $\mathrm{HgCdTe}$ absorber and doped Si thermistor. Because of a drastic rise of thermal boundary resistance (Kapitza resistance) at LT, a detector integrating the functions of absorber and thermometer in a one unit is more attractive. Unfortunately, both $\mathrm{Ge}$ and $\mathrm{Si}$, which are commonly used as bulk thermistors, have low atomic number and thus couldn't be effectively used as integrated detector. We are developing the new superdiluted magnetic semiconductor InSb:Mn with the goal of its application to cryogenic detection particularly in a low-energy range upon utilising large nuclear charge $Z$ of both In and $\mathrm{Sb}$. 


\section{RESULTS}

It is well known that the resistivity of the properly doped $\mathrm{Ge}, \mathrm{Si}$ and many other semiconductors varies at subhelium temperature as $\rho=\rho_{0} \exp \left(\mathrm{T}_{0} / \mathrm{T}\right)^{\mathrm{x}}$, where $1 / 4 \leq \mathrm{x} \leq 1 / 2$ and corresponds to various types of hopping conductivity. At higher temperatures the resistivity obeys $x=1$ law attributed to a gap-controlled conductivity. The thermosensitivity of thermistors is usually defined as $\alpha=R^{-1}(d R / d T)$. Therefore one has $\alpha(T)=-(\mathrm{x} / \mathrm{T})\left(\mathrm{T}_{0} / \mathrm{T}\right)^{\mathrm{x}}$. Because of great importance of $\alpha$ for cryodetector operation, thermistors with most high values of $\alpha$ are desired. From the other hand increasing of $\alpha$ through deacreasing of temperature is limited by a rapidly increasing microphonic noise and the maximal value of $R$ consistent with the read-out electronics. Briefly saying, the difficulties (and prices) rise exponentially below, say $50 \mathrm{mK}$, while dilution refrigerators provide operation down to $2 \mathrm{mK}$.

To avoid technological problems in production of superiow temperature thermometers a giant positive magnetoresistance observed in heavely $\mathrm{Ga}$ doped Ge have been used to tune the thermistor in applied magnetic field [4]. The results were rather poor and show that magnetic field tuning of doped Ge isn't competable against the use of NTD Ge.

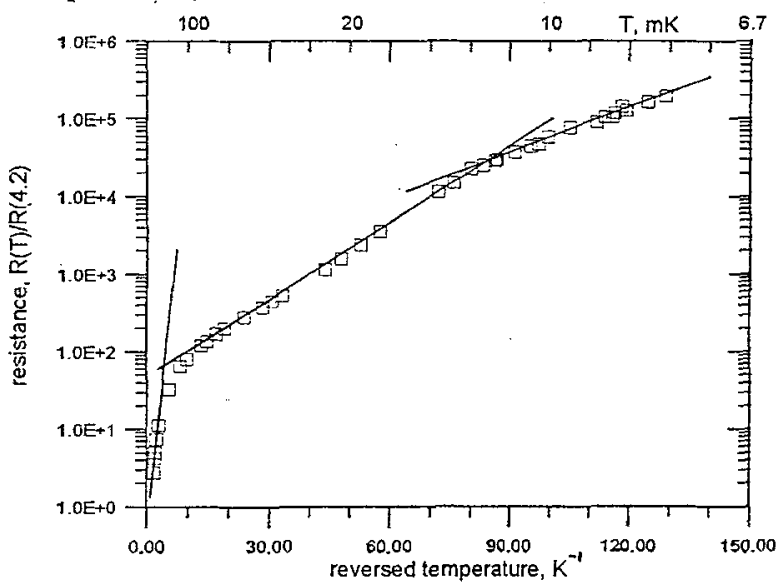

Fig. 1. Temperature dependence of resistivity (symbols) of one pInSb:Mn sample (manganese concentration is $2.14 \times 10^{17} \mathrm{~cm}^{-3}$ ). Three temperature ranges with different activation energies are clearly pronounced.
Earlier we have found that LT conductivity in InSb doped with $M n$ exhibits besides $x=1 / 2$, $\mathrm{x}=1$ behaviour as well [5]. Fig. 1 depicts the exact exponential resistivity of one of the samples down to $7 \mathrm{mK}$. To our knowledge, InSb:Mn is the first type of doped semiconducting thermistors demonstrating such conductivity below $100 \mathrm{mK}$. At Fig.2 R(T) curves for a few types of LT thermistors, NTD Ge, carbon Speer and two InSb:Mn (correspondig to $\mathrm{x}=1 / 2$ and $\mathrm{x}=1$ ) are shown for comparizon. The corresponding alphas are plotted at Fig.3. Two principal advantages of InSb:Mn follows at the first sight, particularly with temperature decreasing: a higher sensitivity $\alpha$, especially for $x=1$ sample, and a few orders higher, compared with NTD Ge, conductivity. The latter is very favourable to the fast response time of detectors and their immunity to microphonic noise. These results motivated further investigations.
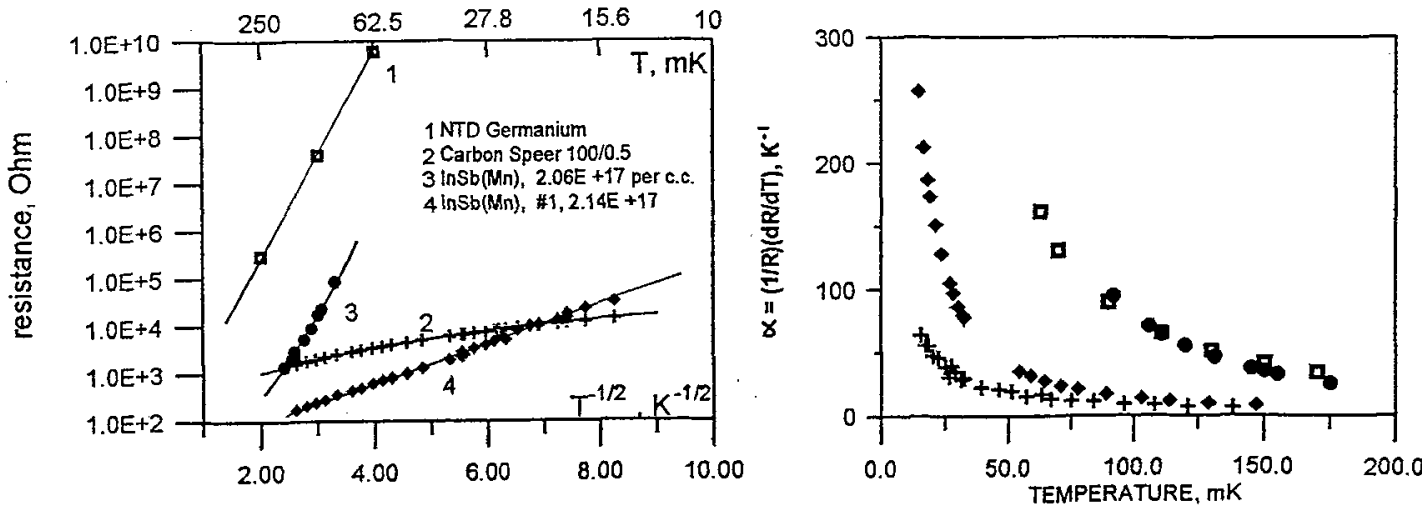

Fig.2. (left). Resistance versus temperature for three types of $\mathrm{LT}$ thermistors. Two samples of InSb:Mn with exponents $\mathrm{x}=1 / 2$ and $\mathrm{x}=1$ are shown.

Fig.3. (right). Thermosensitivity of the same thermistors. InSb with $x=1$ overcomes NTD Ge below $110 \mathrm{mK}$. 


\subsection{Resistivity}

Czochralsky grown and melt doped with manganese InSb crystals were cutted into rectangular samples with a few mms typical size. As is known, Mn produces in InSb the shallow ( $7 \mathrm{meV}$ ) acceptors. Partly compensated with $\mathrm{Te}$ samples were also used. The concentration of the holes have been determined through Hall effect at nitrogen temperature. The $\mathrm{R}(\mathrm{T})$ curves were measured in a dilution refrigerator providing $7 \mathrm{mK}$ minimal temperature. AC bridge with an upper limit on measured resistance of $10^{5} \mathrm{Ohm}$ have been used and the samples were directly immersed into He3-He4 solution in a mixing chamber. Magnetoresistance was studied in a field produced by superconducting magnet.

It was found that the desired conductivity $(x=1$ and convenient $R$ values ) below $200 \mathrm{mK}$ can be obtained for narrow concentration range of $\mathrm{Mn}$ slightly less than the critical concentration of Mott's transition, determined to be $\mathrm{N}_{\mathrm{Mn}}=(2.1+\delta) \times 10^{17} \mathrm{~cm}^{-3}$, the lower temperature range, the more is this concentration range restricted. Taking into account that both resistance and magnetic susceptibility measurements demonstrated an evidence of considerable nonuniformity in dopant distribution, it is clear that the same technological problems as for other semiconductors applied to LT thermometry arise. Fortunately, a giant negative magnetoresistivity (GNM) observed in InSb:Mn permits to avoid these difficulties elegantly. Fig. 4 illustrates GNM in one compensated sample. As is seen, the applied field preserves the temperature dependence of $R$ corresponding to $x=1$ and extended the measurable range of $R$ to lower temperatures. Fig. 5 shows the dependence of
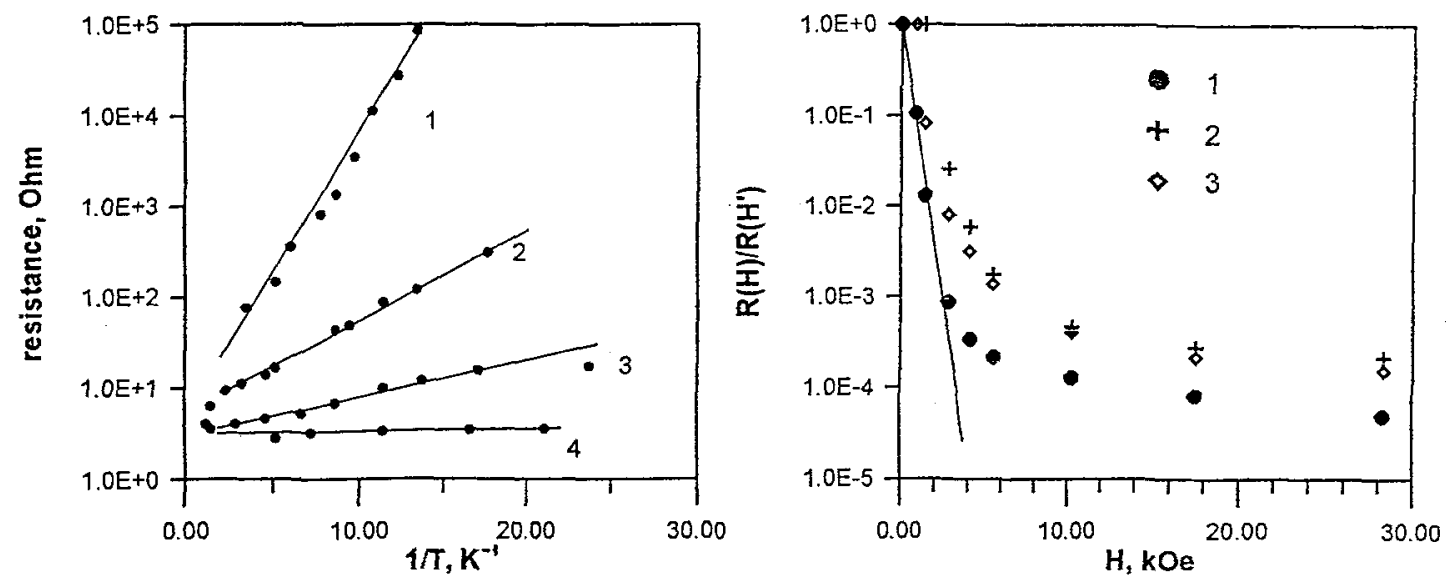

Fig.4. (left). R(T-1) curves for InSb:Mn thermistor with $\mathrm{NMn}_{\mathrm{M}}-\mathrm{N}_{\mathrm{d}}=1.8 \times 10^{17} \mathrm{~cm}^{-3}$ plotted at different values of applied magnetic field.

Fig.5. (right). Negative magnetoresistance in uncompensated InSb:Mn versus applied field at $80 \mathrm{mK}^{1} 1, \mathrm{NMn}^{-1.8 \times 10^{17}} \mathrm{~cm}-3$; 2. $\mathrm{NMn}_{\mathrm{Mn}}=1.6 \times 10^{17} \mathrm{~cm}^{-3} ; 3, \mathrm{NMn}=1.13 \times 10^{17} \mathrm{~cm}^{-3}$.

GNM on applied field at fixed temperature for three uncompensated samples with different Mn concentration. Note, that the reduced values of resistance are taken at different values of field $\mathrm{H}^{\prime}: \mathrm{H}^{\prime}=0$ for sample $1, H^{\prime}=0.83 \mathrm{kOe}$ for sample $2, \mathrm{H}^{\prime}=1.39 \mathrm{kOe}$. for sample 3 . The reason is that the value of resistance for the latter two thermistors in field below $\mathrm{H}^{\prime}$ is above the upper limit of our bridge. A very important practical conclusion is that magnetic field as low as $1 \mathrm{kOe}$ permits one to use unexpensive InSb:Mn thermistors with the thermometric properties better than that of NTD Ge which need a sophisticated technology of production! Though from the application point of view these results are sufficient, the mechanism of LT conductivity in InSb:Mn is very interesting by itself and may be a subject of the special theoretical treatment. Here we would like to restrict ourselves to the note, that undoubtedly this 
mechanism is controlled by magnetic properties of Mn ions. From Fig. 5 it follows that at the temperatures of interest the field influence (weak fields before saturation of $\mathrm{R}$ ) can be accounted in the following form:

$$
\mathrm{R}(\mathrm{T}, \mathrm{H})=\mathrm{R}_{0} \exp ((\Delta-\mathrm{mH}) / \mathrm{kT})
$$

where $\Delta$ is some activation energy (energy gap), and $\mathrm{m}$ is a temperature and field independent coefficient. For the sample manifesting GNM shown in Fig. 4 , for instance, $\Delta=6.75 \cdot 10^{-5} \mathrm{eV}$ at zero field. Getting now the physical meaning of $\Delta$ apart, we would like to make some conclusion aboute GNM, based on equation (1). Fitting of curves in Fig. 5 according to formula (1) gives $m$ close to Bohr magneton $\mu_{\mathrm{B}}$. This enables a simple interpretation if to compare the binding energy of the antiferromagnetically coupled through sp-d exchange interaction hole with spin $S_{h}=1 / 2$ and manganese ion with spin $S_{i}=5 / 2$ in zero and apllied field. Because the exchange interaction can easily be estimated and occurs to be larger then the zeeman energy of $S_{h}$ in a field below $10 \mathrm{kOe}$ and for concentrations of $\mathrm{Mn}$ larger than few units multiplied by $10^{16} \mathrm{~cm}^{-3}$, then in applied field the magnetic moment of $d$-shell will be aligned parallel to field, while moment of the hole coupled to it- contrary to field with excess energy of $\mathrm{g}_{\mathrm{B}} \mathrm{m}_{\mathrm{S}} \mathrm{H}$, where $\mathrm{g}$ is a $\mathrm{g}$-factor of a hole (approximately 2), $\mathrm{m}_{\mathrm{S}}$ is a magnetic quantum number and is equal to $1 / 2$. This lowers the ionization energy of the acceptor. More exact treatment should include the Zeeman splitting of magnetic levels of the acceptor, which slightly change the above estimated values of characteristic energies, but not the physical meaning.

\subsection{Static magnetic susceptibility}

The involvement of exchange interaction into unusial transport properties of superdiluted magnetic semiconductor InSb:Mn at LT seems very likely. In addition to the mentioned earlier antiferromagnetic coupling of acceptor and hole the similar direct and indirect interaction between acceptors in principle is possible, which can result in a long-range magnetic order arizing at low enough temperature. The presence of exchange interection between Mn ions may manifest itself, among all, as a deviation from the Kurie law for magnetic susceptibility. We have measured the latter for uncompensated (Hall determined concentration of $\mathrm{Mn}$ is $2.04 \cdot 10^{17} \mathrm{~cm}^{-3}$ ), and compensated ( $k=0.7$ ) samples in SQUID susceptometer. To derive the paramagnetic addition to the total susceptibility the diamagnetic lattice background was determined at high temperature and subtracted from the data. The measured diamagnetic susceptibility was the same for all the samples and equal to $-1.8 \cdot 10^{-5}$ (SI units) which differs $8 \%$ from the published value

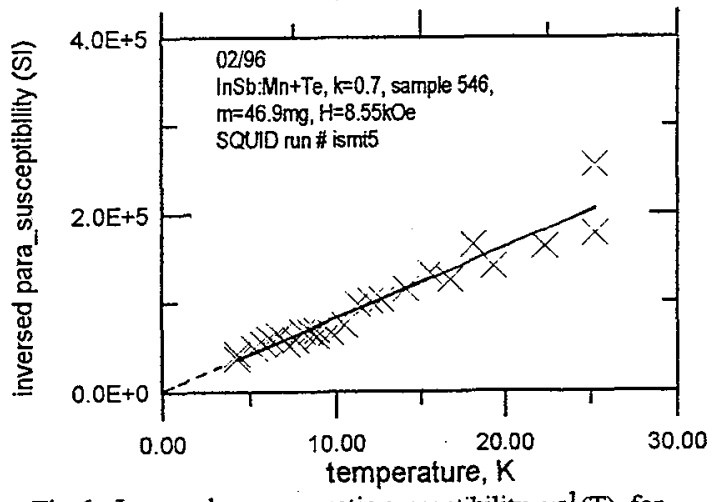

Fig.6. Inversed paramagnetic susceptibility, $\chi^{-1}(T)$, for compensated sample. Straight line is a linear fit which corresponds to concentration $1.4 \times 10^{18} \mathrm{~cm}^{-3}$ of spins $\mathrm{S}=5 / 2$.
[6]. At Fig.6 the inversed paramagnetic susceptibility of compensated sample versus temperature is plotted (symbols). Solid line is a linear fit of measured data excluding origin. An exact Curie law is demonstrated with concentration of $S=5 / 2$ spins equal to 1.4. $10^{18} \mathrm{~cm}^{-3}$. Therefore no presence of $\mathrm{Mn}-\mathrm{Mn}$ interaction is seen. In the case of uncompensated sample the treatment of the data was complicated by a small and rapidly decreasing upon temperature increase value of paramagnetic component, which is only $30 \%$ of the total at the lowest possible temperature of $4.2 \mathrm{~K}$, and, what is more serious, by nonuniform magnetization which strongly deteriorates the accuracy of evaluation of the moment. Thus, unfortunately, we are not able now to make a conclusion regarding neither presence, nor absence of the $\mathrm{Mn}-\mathrm{Mn}$ exchange interaction in uncompensated sample with Mn concentration less than the critical Mott's value. As for the compensated one, our result (no exchange addition to Curie law) is consistent with the earlier published data [7], but it should be noted that in these samples the concentration of $\mathrm{Mn}$ (uncompensated samples) or 
$\mathrm{N}_{\mathrm{Mn}}-\mathrm{N}_{\mathrm{d}}$ (compensated ones) was higher than the critical value of metalization the impurity band, which can strongly affect the magnetic ordering. We are going to repeat the measurements with the more uniform samples and doping level below semiconductor-metal transition

\section{COMPOSITE CRYODETECTOR WITH InSb:Mn THERMISTOR}

The feasibility of InSb;Mn thermometers to detect nuclear particles have been tested with a prototype of a composite cryodetector mounted at the bottom of the mixing chamber in the inner vacuum space of the refrigerator and whose schematic view is shown at Fig.7. The absorber is a $30 \mathrm{~cm}^{3}$-volume and cylindershaped Si crystal. The thermistor with ac $1 \mathrm{~mm}^{3}$ volume was glued to one of the faces of the absorber and was
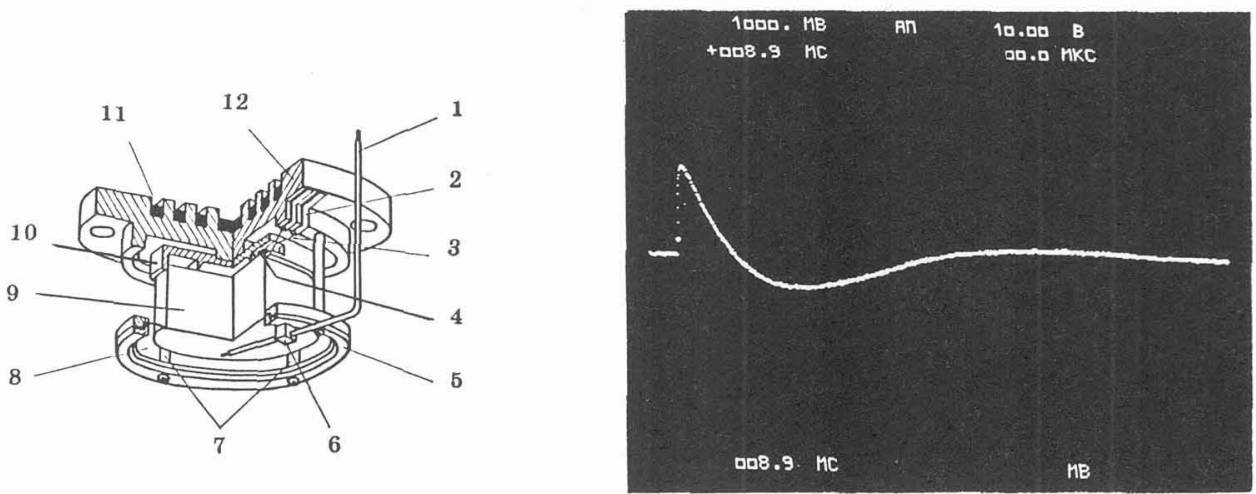

Fig.7. (left). Composite detector housing. 1-high voltage supply; 2-thermal anchor of leads; 3-0,05mm constantan leads; 4InSb:Mn thermistor; 5-copper double ring; 6-teflon holder; 7-copper supports; 8-mylar membrane; 9-30cm3 Si absorber; 10 caprolon supporting frame; 11-sintered copper powder; 12-copper bottom of mixing chamber.

Fig.8. (right). A photograph of a signal produced by cosmic muon and recorded by the composite detector at $70 \mathrm{mK}$. Released energy is $6 \mathrm{Mev}$, trace duration is $0.4 \mathrm{Is}$.

biased from DC battery through $0.05 \mathrm{~mm}$ constantan wires thermally anchored to the mixing chamber. The thermistor and a front-end JFET of the preamplifier placed inside the cryostat are connected by a coaxial line offering low both thermoconductivity and signal loss. The signals were amplified 5000 times and monitired by a digital storage oscilloscope. The operating temperature was $70 \mathrm{mK}$ with corresponding resistance of the thermistor close to2MOhm at 50nA bias. No tuning by magnetic field have been used. Fig. 8 displays an example of the sygnal induced in absorber by a cosmic muon. Time duration of the trace is $0.41 \mathrm{~s}$.

\section{CONCLUSION}

We have prooved experimentally the feasibility of the new-type bulk semiconductor InSb:Mn for nuclear cryodetection. These thermistors offer higher both thermosensitivity and conductivity over NTD Ge and can be used at most LT obtained in dilution cryostats. They exhibit giant negative magnetoresistance effect which enables one to match the properties of the thermistor to desired range by applying of a weak magnetic field $(1 \mathrm{kOe})$, independent of the doping accuracy and nonuniform distribution of $\mathrm{Mn}$ and, therefore, to overcome the main technological difficulties imminent to application of the doped $\mathrm{Ge}$ and $\mathrm{Si}$ at superlow temperature. To our opinion the most attractive areas of application are nuclear spectroscopy for which small-volume detectors are enough (low-energy X-ray and beta's, $\alpha$ 's), and, probably, electromagnetic radiation quantum detectors with tuned spectral sensitivity, according to eq.(1). 


\section{References}

[1] Proc. 6th Workshop on Low Temperature Detectors, LTD-6, Beatenberg, Switzerland, Sept. 1995, To be published in Nucl Instrum. and Methods.

[2] McCammon D. et al., Nuclear Physics A527 (1991) 821c-824c.

[3] McCamon D. et al., 3rd Int. Workshop on Low Temperature Detectors for Neutrinos and Dark Matter, LTD-3, Gran-Sasso, Italy, 1989, ed. by L.Brogiato, D.Camin and E.Fiorini, Editions Frontieres, 1990, p. 213.

[4] De Moor P. et.al., J.of Low Temp.Physics 93 (1993) 295 and references therein.

[5] Obukhov S.A. et al., Cryogenics 31 (1991) 874.

[6] Bowers R. and Yafet Y., Phys.Rev. 115 (1959) 1165.

[7] Andrianov D.G. et al., Physics and Technics of Semiconductors 9 (1975) 1555 (in Russian). 